\title{
Effect of the Dot-Dot Interaction Strength on the Conductance of Side-Connected Quantum Dots
}

\author{
Maria A. Davidovich, V. M. Apel, and E. V. Anda \\ Departamento de Física, Pontifícia Universidade Católica do Rio de Janeiro, 22453-900, Brazil
}

Received on 8 December, 2005

\begin{abstract}
The conductance of two interacting dots connected to leads is studied. The configuration is such that one dot is embedded into the leads while the other is tunneling-coupled only to the first dot. The effect of the tunneling interaction strength on the conductance is discussed. As the two dot levels cross the Fermi level the low temperature conductance of the system cancels out, due to interference effects. This cancellation persists over a range of gate potential that depends upon the interaction strength: the greater the interaction the larger the range of gate potential where the current vanishes.
\end{abstract}

Keywords: Double-dot; Conductance; Kondo effect

\section{INTRODUCTION}

Since the first observation of the Kondo effect in a single quantum dot[1] several single and double quantum dot devices have been studied theoretically[2] and experimentally[3]. In the last years interest in interacting double-quantum-dot systems has been renewed due to their potential applications to quantum computing[4]. The strength of the interaction between the two quantum dots determines the character of the electronic states and the nature of the transport through them. In the limit of weak tunneling interaction the electrons are localized on the individual dots while in the strong limit the delocalized electronic charge is no longer quantized. The transport properties depend also on the topology of the double-dot system[5]. For the case of the two dots aligned with the leads, in the weak coupling limit the interaction of each dot with the conduction electrons of the nearby lead gives rise to the Kondo in both dots. In the opposite limit, the strong interdot tunneling interaction together with the intra-dot Coulomb repulsion result in an anti-parallel alignment of the two dot spins and the Kondo effect is suppressed. By increasing the tunneling interaction the dot system goes continuously from the Kondo regime to an antiferromagnetic state of the dots. Another system of two-interacting dots in the side-connected configuration has special interest since it permits the control of the current along the leads by changing the state of charge of the side-connected dot. The conductance of this system has been studied as a function of the gate potentials applied to the dots, using the embedded cluster method[5] and the numerical renormalization group technique[6]. In these two works the $\mathrm{T}=0$ dependence of the conductance on the gate potential applied to the dots present different behaviors. In this paper we use the equation of motion method to analyze this system. We discuss, in particular, the dependence of the conduction upon the gate voltage as the dot-dot tunneling interaction strength is increased. The temperature dependence of the conduction is also obtained. In order to make the method more tractable we restrict our calculation to the case of infinite Coulomb repulsion U.

\section{THEORY}

An Anderson two-impurity first neighbor tight binding Hamiltonian represents the system. Naming QD1 the inserted dot and QD2 the side-connected one, the total Hamiltonian reads,

$$
\begin{aligned}
H & =\sum_{\substack{i=1,2 \\
\sigma}}\left(V_{i}+\frac{U_{i}}{2} n_{i \sigma}\right) n_{i \sigma}+t_{12} \sum_{\sigma}\left(c_{1 \sigma}^{+} c_{2 \sigma}+c_{2 \sigma}^{+} c_{1 \sigma}\right) \\
& +\sum_{k r \sigma}\left[t_{r}\left(c_{k r \sigma}^{+} c_{1 \sigma}+c_{1 \sigma}^{+} c_{k r \sigma}\right]+\sum_{k r \sigma} \varepsilon_{k r} c_{k r \sigma}^{+} c_{k r \sigma}\right.
\end{aligned}
$$

The first two terms represent the Hamiltonian of the doubledot with dot energy levels $V_{i}(i=1,2)$, intra-dot Coulomb interaction $U_{i}$ and the inter-dot hopping interaction $t_{12}$. The third term describes the coupling of QD1 to the right and left contacts through the hopping interaction $t_{R}$ and $t_{L}$, respectively, that we assume to be independent of the conduction electron wave vector $k$. The last term describes the conduction electrons of the contacts with energy $\varepsilon_{k}$ for both left and right contacts, whereas $c_{k r \sigma}^{+}$and $c_{k r \sigma}$ are, respectively, the creation and annihilation operators for the conduction electrons in contact $r(r=L, R)$, with wave vector $k$, and spin $\sigma$.

The Green functions for the system are obtained by the equation of motion method in a decoupling scheme that incorporates the phenomena associated to the Kondo effect. This approximation permits the study of the temperature dependence of the properties of the system. Although it is reliable for $T<T_{K}$, the limit $\mathrm{T}=0$ is not adequately treated, as discussed below. Frequency dependent equations for the Green functions related to the two dots, $G_{11}, G_{22}$ and $G_{12}$, must be solved. The equation for the Green function at QD1, is given by:

$$
\begin{aligned}
\left(\omega-\varepsilon_{1}\right) G_{11}^{\sigma}= & 1+t_{S} \sum_{k r}\left\langle\left\langle c_{k r \sigma} ; d_{1 \sigma}^{\dagger}\right\rangle\right\rangle+t_{12}\left\langle\left\langle d_{2 \sigma}^{\dagger} d_{1 \sigma}^{\dagger}\right\rangle\right\rangle \\
& +U\left\langle\left\langle d_{1 \bar{\sigma}} ; d_{1 \sigma}^{\dagger}\right\rangle\right\rangle
\end{aligned}
$$

where $G_{11}^{\sigma}=<<d_{1 \sigma} ; d_{1 \sigma}^{\dagger}>>, d_{1}^{\dagger}\left(d_{1}\right)$ is the creation (annihilation) operator for electrons in QD1 and the interaction of this dot with the two leads has been assumed to be equal, $t_{s}=t_{R}=t_{L}$. Similar equations can be written for the other Green functions. These functions are expressed in terms of higher order Green functions, constituting an infinite chain that has to be truncated at some point. Keeping only terms 
proportional to $t_{12}^{2}$ and $t_{s}^{2}$, and considering a very strong intradot Coulomb interaction $U$ we arrive, after some manipulations, at the following expressions for $G_{11}, G_{22}$ and $G_{12}$ :

$$
\begin{gathered}
G_{11}^{\sigma}=\frac{\left(1-\left\langle n_{1 \bar{\sigma}}\right\rangle\right)\left(\omega-\varepsilon_{2}\right)-t_{12}\left\langle d_{1 \bar{\sigma}}^{\dagger} d_{2 \bar{\sigma}}\right\rangle}{\left(\omega-\varepsilon_{2}\right)\left(\omega-\varepsilon_{1}-t_{s}^{2} \sum_{k r} \frac{1+\left\langle n_{k r \bar{\sigma}}\right\rangle}{\omega-\varepsilon_{k}}\right)-t_{12}^{2}} \\
G_{22}^{\sigma}=\frac{\left\langle 1-n_{2 \bar{\sigma}}\right\rangle\left(\omega-\varepsilon_{1}-t_{s}^{2} \sum_{k r} \frac{1+\left\langle n_{k r \bar{\sigma}}\right\rangle}{\omega-\varepsilon_{k}}\right)-t_{12}\left\langle d_{1 \bar{\sigma}}^{\dagger} d_{2 \bar{\sigma}}\right\rangle}{\left(\omega-\varepsilon_{2}\right)\left(\omega-\varepsilon_{1}\right)-\left(\omega-\varepsilon_{2}\right) t_{s}^{2} \sum_{k} \frac{1+\left\langle n_{k r \bar{\sigma}}\right\rangle}{\omega-\varepsilon_{k}}-t_{12}^{2}} \\
G_{21}^{\sigma}=\frac{\left(\left\langle 1-n_{2 \bar{\sigma}}\right\rangle\left\langle 1-n_{1 \bar{\sigma}}\right\rangle-\left\langle d_{1 \bar{\sigma}}^{\dagger} d_{2 \bar{\sigma}}\right\rangle\right) t_{12}}{\left(\omega-\varepsilon_{2}\right)\left(\omega-\varepsilon_{1}\right)-\left(\omega-\varepsilon_{2}\right) t_{s}^{2} \sum_{k r} \frac{1+\left\langle n_{k r \bar{\sigma}}\right\rangle}{\omega-\varepsilon_{k}}-t_{12}^{2}}
\end{gathered}
$$

where $\varepsilon_{i}(\mathrm{i}=1,2)$ is the gate potential at QDi and $n_{k r \bar{\sigma}}$ is the occupation number for electrons with wave vector $\mathrm{k}$ and spin $\sigma$ at the left $(r=L)$ and right $(r=R)$ contacts.

Notice that the QD1 and QD2 occupation numbers, $\left\langle n_{1}\right\rangle$ and $\left\langle n_{2}\right\rangle$ in Eqs. (3) and (4), are obtained by the imaginary part of the $G_{11}$ and $G_{22}$, respectively, so that the solution has to be obtained self-consistently. Moreover, $G_{11}$ and $G_{22}$ depend also on the average value $\left\langle d_{1}^{\dagger} d_{2}\right\rangle$ that is obtained by the imaginary part of the Green function $G_{12}$. Therefore a selfconsistent calculation involving the three Green functions simultaneoulsy has to be done.

At finite temperature the conductance $G$ and the charge occupation $n_{i}$ at QDi are calculated self-consistently, using the following expressions:

$$
G=\frac{2 e^{2}}{h} t_{s}^{4} \int_{-\infty}^{\infty}\left|G_{11}^{\sigma}\right|^{2} \rho^{2}(\omega) \frac{\partial f(\omega)}{\partial \omega} d \omega
$$

and

$$
n_{i \sigma}=-\frac{1}{\pi} \int_{-\infty}^{\infty} \operatorname{Im}\left(G_{i i}^{\sigma}\right) f(\omega) d \omega
$$

where $\rho(\omega)$ is the lead density of states that we consider constant, a good approximation for states near the Fermi level, and $f(\omega)$ is the Fermi distribution function.

\section{RESULTS}

The Fermi level is taken at $\varepsilon_{F}=0$ and the interaction of QD1 with the right and left contacts is considered equal, $t_{R}=t_{L}=t_{s}$. All energies are in units of $\Gamma=t_{s}^{2} / W$, where $\mathrm{W}$ is the lead bandwidth. For simplicity we restrict our analysis to the case of identical dots, $V_{1}=V_{2}=V$. As can be concluded from a detailed inspection of the previous equations for the Green functions, at $T=0$ the discontinuity of the Fermi distribution function creates a logarithmic singularity at the Fermi level that is an artifact of the decoupling procedure adopted to calculate the Green Functions. The problem is solved by increasing the temperature above a characteristic value $T_{m}$ that

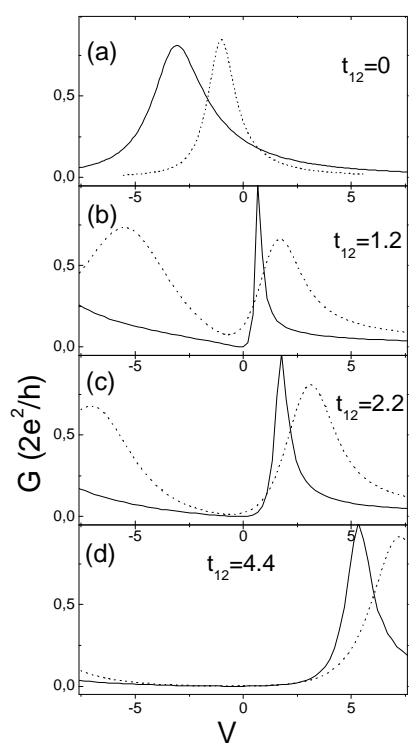

FIG. 1: Conductance in units of $2 e^{2} / h$ as a function of the gate potential at the dots $V=V_{1}=V_{2}$ for the dot-dot interaction $t_{12}=$ 0,0.2, 1.2, 2.2 and 4.4; low $\mathrm{T}$ (continuous lines) and high $\mathrm{T}$ (dotted lines).

eliminates the logarithm singularity. This is not a serious restriction to study the Kondo regime because at a particular value of the gate potential the Kondo temperature $T_{K}$ satisfies the condition $T_{K}>T m$. However, as we would like to study the evolution of the system as a function of the gate potential for a fixed value of the temperature, we have to adopt the more restrictive condition for $\mathrm{Tm}$ that corresponds to the situation when the system is entering into the Kondo regime $\left(V_{1} \sim \Gamma\right)$. This implies that, as the gate potential and, consequently, the Kondo temperature is reduced, the system is driven out of the Kondo regime since $T_{K}<T_{m}$. Our results for the conductance shown below reflect this fact. Anyhow, this represents, to a large extent, the real situation as measurements are always done at finite temperatures.

In Figs. 1 and 2 we display the conductance and the dot charges as a function of $\mathrm{V}$, for different values of the interaction strength $t_{12}$. Let's first discuss the conductance results, where the continuous lines correspond to low temperature $\left.\left(T_{L}=2 \times 10^{-4}\right)\right)$ and the dashed ones to high temperature $\left(T_{H}=2 \times 10^{-1}\right)$. Fig.1(a), for $t_{12}=0$, represents the one-dot conductance. As the gate potential decreases from positive values and passes through the Fermi level, QD1 approaches the resonance condition and enters in the Kondo regime permitting the electrons to go along the system. As $V$ is further diminished the current diminishes because the Kondo resonance disappears since, at these values of gate potential, the Kondo temperature $T_{K}$ is smaller than $T_{m}$, as discussed above. The result for high $\mathrm{T}$ reproduces the well known Coulomb blockade peak. Note that, since we are not at $T=0$ the low $\mathrm{T}$ one-dot conductance does not reach the maximum value $2 e^{2} / h$.

In the rest of the Fig. 1 the conductance for increasing interdot interaction $t_{12}$ is depicted. In all cases by decreasing the 


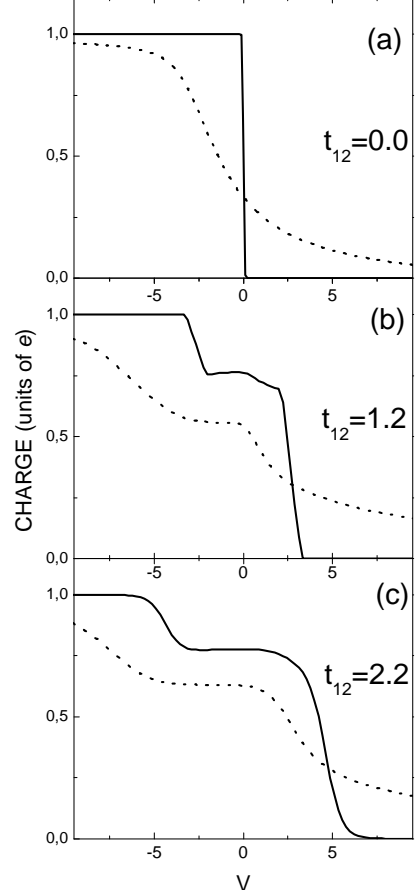

FIG. 2: Charge at QD2 (continuous lines) and QD1 (dotted lines) as a function of the gate potential at the dots $V=V_{1}=V_{2}$ for low $\mathrm{T}$ and $t_{12}=0,1.2$ and 2.2 .

gate potential the conduction increases as soon as charge begins to enter into the embedded dot, offering a path for the electrons to flow along the system. As the gate potential is further reduced, the side-connected dot gets abruptly charged and interferes with the partially charged embedded dot resulting in the cancellation of the conductance. This is due to the existence of two paths for the electrons to go through the double-dot system. The conductance peak presents a Fano anti-resonance shape [7]. For high temperature (dotted lines) this phenomenon is restricted to the resonance condition although smeared out due to thermal effects. Although the origin of the cancellation of the current in both cases is the same, the shape of the $G \times V$ curves differ due to the fact that, at low $\mathrm{T}$, the two dots are in the Kondo state. This has been demonstrated by the analysis of the low $\mathrm{T}$ spectral density that shows Kondo peaks related to the two dots that disappear as the tem- perature increases. These results will be discussed in more detail elsewhere.

The range of gate potential where the current vanishes gets more extended as the interaction increases, as shown in Figs. 1 (c),(d) and (e). This is expected since the energy spacing between the peaks is approximately proportional to $t_{12}$. Current cancellation at the Fermi level has also been obtained for this same system in a calculation using the embedded cluster method[5].

The charges at each quantum dot, displayed in Fig. 2, correspond to low temperature, $T_{L}$. The high $\mathrm{T}$ charge behavior does not differ much from the low $\mathrm{T}$ and is not shown. For disconnected dots, (Fig. 2(a)), charge enters continuously into QD1 and abruptly into QD2, at the Fermi level, as expected since this dot is isolated from the contacts. When the dots are weakly connected, the QD2 charge reaches the value of one electron going through two jumps, at the values of gate potential such that the current begins to flow along the system. In this situation QD2 interacts weakly with the lead conducting electrons. As the dot-dot interaction increases the charge jumps get smoother. In this case the states of the double-dot are a combination of the two dot sates, and the charging of two dots are is similar (Fig. 2c). In any situation QD1 charge increases continuously since it is directly connected to the lead conduction electrons.

\section{CONCLUSIONS}

We have studied the conductance of a double quantum dot device in which one of the dots is embedded into conducting leads while the other is connected only to the first dot through a tunneling interaction. The zero temperature conductance of the system vanishes at gate potential values around the Fermi level. This is due to interference effects caused by the possibility of the electrons to go through the dots using two paths. The range of gate potential values for which the current vanishes increases with the tunneling dot-dot interaction strength.

\section{Acknowledgments}

We acknowledge support from the Brazilian Agencies FAPERJ, CNPq and CAPES.
[1] D. Goldhaber-Gordon, H. Shtrikmann, D. Mahalu, D. AbuschMagder, U. Meirav, and M. A. Kastner, Nature 391, 156 (1998).

[2] A. Georges and Y. Meir, Phys. Rev. Lett. 82, 3508 (1999).

[3] J. C. Cheng, A. M. Cheng, and M. R. Melloch, Phys. Rev. Lett. 92, 176801 (2004); W.G.van der Wiel, S. D. Franceschi, J. M. Elzerman, T. Fujisawa, S. Tarucha, and L. P. Kouwenhove, Rev. Mod. Phys. 75, 1 (2003); J.M. Elzerman, R. Hanson, J. S. Greidanus, L. H. Willems van Beveren, S. De Franceschi, L. M. K. Vandersypen, S. Tarucha, and L. P. Kouwenhoven, Phys. Rev. B 67, 161308 (2003).
[4] D.P. DiVicenzo Science 720, 255 (1995); G. Burkard, D. Loss, and D. P. DiVicenzo, Phys. Rev. B 59, 2070 (1999); D. Loss and D. P. DiVicenzo, Phys. Rev. A 57, 120 (1998).

[5] V. M. Apel, M. A. Davidovich, E. V. Anda, and G. Chiappe, European Phys. J. B. 40, 365 (2004).

[6] P. S. Cornaglia and D. R. Grempel, Phys. Rev. B 71, 075305 (2005)

[7] J. L. DAmato, H. M. Pastawski, and J. F. Weisz, Phys. Rev. B 39, 3554 (1989). 